\title{
A method for evaluating theoretical and real operation of diesel engines in energy conversion formulation taking into account their operating indices
}

Jerzy Girtler, Prof.

Gdansk University of Technology

\begin{abstract}
The article proposes valuating the operation of an arbitrary diesel engine, based on the sample case of a ship's main engine in which energy conversion processes take place in a given time. The above operation is understood as the energy transfer to the screw propeller in the given time in which the energy conversion into work and/or heat and its further transmission take place. The here proposed method for evaluating the operation of the main engines installed in marine power plants consists in comparing the operation of these engines to a physical quantity the measuring unit of which is the joule-second (joule $\times$ second). A new term is introduced which bears the name of the theoretical engine operation and is the standard (ideal) operation which can be compared to the operation of real engines revealing different levels of wear. It was shown that the calculations of the theoretical operation defined in the above way cannot make direct use of commonly known theoretical Diesel and Sabathe cycles. Instead they should use the cycles modified by heat abstraction taking place in accordance with the isobaric, or isothermal process. Other new terms introduced in the article are: the degree of excellence of energy conversion to work, considered as the measure of excellence of engine operation, and the degree of engine operation dissipation, being the measure of its real operation. It is shown that if in time t of engine operation the case takes place that: $L_{i}=i d e m$ and $L_{e}$ =idem, then the engine operation dissipation is equal to its mechanical efficiency.
\end{abstract}

Key words: operation, energy, diesel engine, ship's main engine

\section{INTRODUCTION}

The engine operation can be interpreted (in a descriptive formulation) as the result of the existence of the engine energy state which enables the engine to convert the energy $\mathrm{E}$ into heat and work in a given time $t[2,6,9,10,13]$. That means that when analysing the operation of these engines we have to take simultaneously into account the energy generated by the engines and the time of its conversion $[6,7]$. In this interpretation the engine operation (in valuating formulation) can be compared to a physical quantity which is described by a numerical value and the measure unit bearing the name of the joule-second [joule $\times$ second]. The engine operation understood in the above way becomes worse when the engine wear increases. This means that the value of the operation of each engine will decrease with time, compared to the operation of the (ideal) reference engine.

Obviously, the energy conversion into heat and work, which is observed in the working spaces of each internal combustion engine, may take place in different times. In practice, it is essential that the work done in a given time is possibly the largest, or the given work is done in the shortest possible time. In is also important in practice that the maximum heat is released during the combustion, while the lost heat is possibly the smallest. If the most favourable energy conversion cannot be achieved, we conclude that the engine is in the partial capability state $[3,4,10,12]$. Consequently, of high importance is the analysis of the engine operation and its comparison with the theoretical operation represented by the (ideal) reference engine operating in accordance with the theoretical cycle. Therefore there is a need for determining the theoretical operation of the ideal engine. An attempt to determine such theoretical operation will be presented using the marine engine as the sample case $[6,7]$, but it will also refer to other piston internal combustion engines.

\section{MAIN ENGINE OPERATION IN DETERMINISTIC FORMULATION}

In the deterministic formulation, the operation of the main engine can be analysed in two cases. In the first case we assume that the energy $\mathrm{E}$ transmitted by the engine to the screw propeller does not change in the time interval $[0, t]$. In that case we can assume that the engine operation (D) can be given by the formula:

$$
\mathrm{D}=\mathrm{Et}
$$

In the second case, when the energy (E) transmitted by the engine to the screw propeller depends on time $(\mathrm{t})$, the operation $\mathrm{D}(\mathrm{t})$ is to be calculated using the formula: 


$$
\mathrm{D}(\mathrm{t})=\mathrm{D}=\int_{0}^{\mathrm{t}} \mathrm{E}(\tau) \mathrm{d} \tau
$$

E - energy converted and transmitted during engine operation,

$[0, t]$ - time interval of engine operation.

The energy is released in the form of work or heat. Therefore, according to formula (2) the engine operation can be interpreted as [4]:

$$
\mathrm{D}_{\mathrm{L}}=\int_{0}^{\mathrm{t}} \mathrm{L}(\tau) \mathrm{d} \tau ; \mathrm{D}_{\mathrm{Q}}=\int_{0}^{\mathrm{t}} \mathrm{Q}(\tau) \mathrm{d} \tau
$$

where:

$\mathrm{D}_{\mathrm{L}}$ - operation connected with the performed work (L),

$\mathrm{D}_{\mathrm{Q}}$ - operation connected with the heat transfer $(\mathrm{Q})$,

$\left[\mathrm{t}_{1}, \mathrm{t}_{2}\right]$ - time interval of engine operation,

$\mathrm{t}$ - time, independent variable.

The conditions of main engine operation are mainly determined by the external conditions in which the sea-going ship sails and tasks done by the ship users (the crew) [8]. These conditions and tasks are the reason why in different time intervals different amounts of energy are converted inside the working spaces of these engines. However, in each case the main engine operation is determined by their performance areas [6, $9,15]$. These areas are limited by their velocity characteristics, such as the external and control characteristics. If these characteristics are mapped against the main engine propeller characteristics, which also belong to the velocity characteristics, then the ranges of operation of these engines are defined, which can be illustrated in the form of areas (Fig. 1).

The real characteristics differ from their theoretical equivalents (Fig. 1) as the average torque $\left(\mathrm{M}_{\mathrm{o}}\right)$ of the piston internal combustion engine is the function of the average effective pressure $\left(\mathrm{p}_{\mathrm{e}}\right)$, i.e.

$$
\mathrm{M}_{\mathrm{o}}=\mathrm{c}_{\mathrm{s}} \mathrm{p}_{\mathrm{e}}
$$

and the effective pressure $\left(\mathrm{p}_{\mathrm{e}}\right)$ depends remarkably not only on the fuel charge, but also on the engine rotational speed (n) $[2,9,13]$ :

$$
p_{e}=f\left(\Delta G_{p}, W_{d}, p_{d}, R_{\mu}, T_{d}, L_{o}, \lambda, \eta_{v}, \eta_{i}, \eta_{m}\right)
$$

where:

$\mathrm{c}_{\mathrm{s}}$ - coefficient determining constructional properties of the engine,

$\Delta \mathrm{G}_{\mathrm{p}}$ - fuel charge,

$\mathrm{W}_{\mathrm{d}}{ }^{\mathrm{p}}$ - (net) calorific value of the fuel,

$\mathrm{p}_{\mathrm{d}} \quad$ - pressure of the air supplied to engine working spaces,

$\mathrm{R}_{\mu} \quad$ - universal (absolute) gas constant $\left(\mathrm{R}_{\mu} \equiv \mathrm{R} \cdot \mathrm{M}\right)$,

$\mathrm{T}_{\mathrm{d}}^{\mu} \quad-$ temperature of the air supplied to the engine working spaces,

$\mathrm{L}_{\mathrm{o}} \quad$ - theoretical amount of air needed for combustion of 1 $\mathrm{kg}$ of fuel,

$\lambda-$ excess air number,

$\eta_{v} \quad-$ efficiency of filling of the engine working space (cylinder),

$\eta_{i} \quad-$ indicated efficiency,

$\eta_{\mathrm{m}}-$ mechanical efficiency of the engine.

The main engine operation is time-limited in the overload area, especially in the torque overload area, the area $9^{*}-3^{*}-4-9-9^{*}$ in the real characteristic (Fig. 1). The engine cannot also operate long in the area of pure speed overload, the area $6-7^{*}-10^{*}-9-6$. Obviously, the engine operation is even more time-limited in

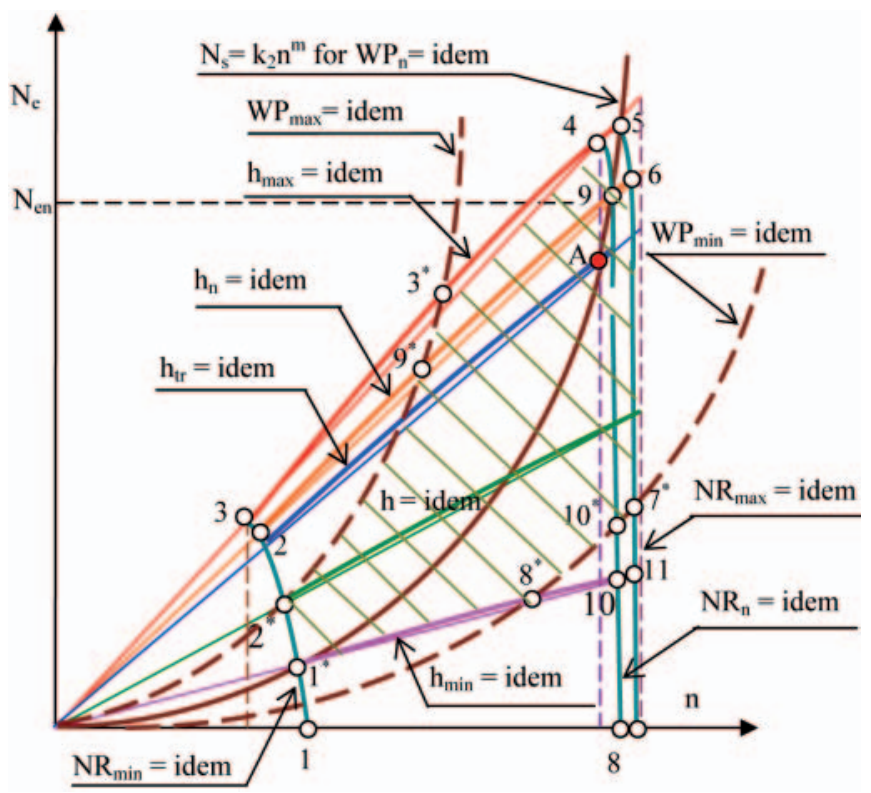

Fig. 1. Speed characteristics of the real piston engine (main engine) installed on a sea-going ship [6]:

- engine performance area (the area of permissible engine loads, the area of so-called stable engine operation): $1^{*}-2^{*}-9^{*}-9-10^{*}-10-8^{*}-1^{*}$, - engine overload area: $2-3-3^{*}-4-5-6-7^{*}-10^{*}-9-2$, - area illustrating the range of engine operation: $1^{*}-2^{*}-9^{*}-3^{*}-4-5-6-7^{*}-10^{*}-8^{*}-1^{*}$,

$\boldsymbol{N}$-effective power, $\boldsymbol{N}$ - nominal (rated) effective power, $\boldsymbol{n}$-rotational speed, $\boldsymbol{h}_{\max }-$ maximal setting of the injection pump, $\boldsymbol{h}_{\boldsymbol{n}}-$ nominal (rated) setting of the injection pump, $\boldsymbol{h}_{t r}$ - continuous setting of the injection pump

$\boldsymbol{h}$ - minimal setting of the injection pump, $\boldsymbol{N} \boldsymbol{R}$ - maximal setting of the controller, $\boldsymbol{N R}_{n}$ - nominal (rated) setting of the controller, $\boldsymbol{N R}_{\text {min }}$ - minimal setting of the controller, $\boldsymbol{W P}_{\max }-$ most difficult sailing conditions (largest resistance of ship motion), $\boldsymbol{W P}_{\boldsymbol{n}}$ - nominal sailing conditions, $\boldsymbol{W P}_{\min }-$ easiest sailing conditions (smallest resistance of ship motion), $\boldsymbol{N}_{s}^{\min }=\boldsymbol{k}_{2} \boldsymbol{n}^{\boldsymbol{m}}-$ real propeller characteristic illustrating the dependence of the power needed by the propeller on the rotational speed (n), $\boldsymbol{k}_{2}$ - real power factor, $\boldsymbol{m}$-coefficient depending on the ship's hull shape (for displacement hulls $m \approx 3.0$ )

the area of both torque and speed overload, the area 4-5-6-9-4 in Fig. 1.

The real performance area (working area) of the engine is limited by the curves: $\mathrm{h}_{\max }=\mathrm{idem}, \mathrm{NR}_{\max }=\mathrm{idem}, \mathrm{h}_{\min }=\mathrm{idem}$ and $\mathrm{NR}_{\min }=$ idem. But the range of the main engine operation is smaller than the performance area, as the main engine can only operate in such a way that it secures delivering the energy needed by the screw propeller for executing a given task. The amount of the energy needed by the propeller in given operating conditions depends on the real propeller characteristic $\mathrm{N}_{\mathrm{s}}=\mathrm{f}\left(\mathrm{k}_{2}, \mathrm{n}\right)$, which is different for different sailing conditions $\mathrm{WP}_{\mathrm{n}}^{\mathrm{s}}=$ idem.

The WP conditions can change between the easiest $\left(\mathrm{WP}_{\min }=\right.$ $=\mathrm{idem})$ and the most difficult $\left(\mathrm{WP}_{\max }=\right.$ idem $)$. These conditions determine the range of main engine operation[5], the area: $1^{*}-2^{*}-9^{*}-3^{*}-4-5-6-7^{*}-10^{*}-8^{*}-1^{*}$ in Fig. 1.

The effective power $\mathrm{N}_{\mathrm{e}}$ generated by the main engine (Fig. 1) includes the information how fast the effective work $\mathrm{L}_{\mathrm{e}}$ will be done in the given time $\mathrm{t}[3,9]$. That means that the power characterises the engine operation in the aspect of the rate of energy conversion to work, taking into account various losses, in particular thermal loss. But the engine operation consisting in the energy conversion into work is not possible if earlier the energy has not been converted to heat in the engine working spaces $[3,4,9,14]$. Therefore when analysing the main engine operation we should take into account the energy delivered to the engine in the fuel-air mixture, which is initially converted to heat (Q) and then to work (L), rather than pure engine power output. 
These considerations show that in case of the main engines installed on ships, as well as all other piston internal combustion engines, converting the chemical energy contained in the fuelair mixture generated in the combustion chambers into heat, and then into the mechanical energy of the crankshaft-piston assembly makes it possible to generate the effective power $\mathrm{N}_{\mathrm{e}}$. This power is to be generated in time t needed for executing a given task by the ship. That means that in order to execute this task in the given time, the effective work $\mathrm{L}_{\mathrm{e}} \cdot \mathrm{t}$ is to be done by an arbitrary main engine. The realisation of this work is the effect of the torque $\left(M_{0}\right)$ generated on the crankshaft at a given rotational speed (n) of each piston internal combustion engine, including the main engine $[2,10,15]$. Therefore according to formula (2), the engine operation interpreted as the energy conversion into effective work $\mathrm{L}_{\mathrm{e}}$ in time $\mathrm{t}$ can be given as:

$$
\mathrm{D}_{\mathrm{L}_{\mathrm{e}}}=\int_{0}^{\mathrm{t}} \mathrm{L}_{\mathrm{e}}(\tau) \mathrm{d} \tau=2 \pi \int_{0}^{\mathrm{t}} \mathrm{n}(\tau) \mathrm{M}_{\mathrm{o}}(\tau) \tau \mathrm{d} \tau
$$

The main engines used on container ships, bulk carriers, and tankers are two-stroke, low-speed engines, most frequently with uniflow scavenging, or lateral scavenging with inlet ports higher than outlet ports $[1,10,14]$. A sample indicator diagram recorded on such an engine is shown in Fig. 2a for the case of uniflow scavenging, and in Fig. $2 \mathrm{~b}$ for the engine with lateral scavenging and inlet ports $\left(h_{d}\right)$ higher than outlet ports $\left(h_{w}\right), h_{d}>h_{w}$.

The presented real cycles show that the compression of the fresh charge (the mixture of the air delivered to the cylinder and the remnants of the exhaust gas which remained in the cylinder after the previous cycle) takes place after the cylinder scavenging process, consisting in the inflow of the fresh air with the simultaneous outflow of the exhaust gas, has ended. Until then, starting from the time instant of removing the exhaust gas from the cylinder, the heat is abstracted with the exhaust gas to the environment. Therefore theoretical cycles of diesel engines should take into account not only isochoric heat abstraction (like in the Diesel and Sabathe cycles), but also, depending on engine speed [7]:

- heat abstraction at constant pressure (isobaric), in case of low-speed engines,

- heat abstraction at constant temperature (isothermal), in case of medium- and high-speed engines.

The above approach is possible if the assumptions are adopted that:

- in low-speed engines the flow resistances change insignificantly and we can assume that the heat abstraction process takes place at the same pressure.

- however, during the realisation of this process remarkable temperature change can be observed due to a relatively long time of heat transfer to the environment (compared to high-speed engines), before the abovementioned fresh charge compression is initiated in the cylinder.

On the other hand, in medium-speed and, especially, highspeed engines such analyses are possible if we assume that:

- the flow resistances change remarkably (compared to low-speed engines) and we cannot assume that the heat abstraction process takes place at constant pressure.

- the temperature change observed during the realisation of this process is neglectable due to a relatively short time of heat transfer to the environment (compared to low-speed engines) before the fresh charge compression starts in the cylinder. Therefore we can assume in that case that after the isochoric heat abstraction is ended, the isothermal or quasi-isothermal heat abstraction process takes place in these engines before the fresh charge compression starts in the cylinder.
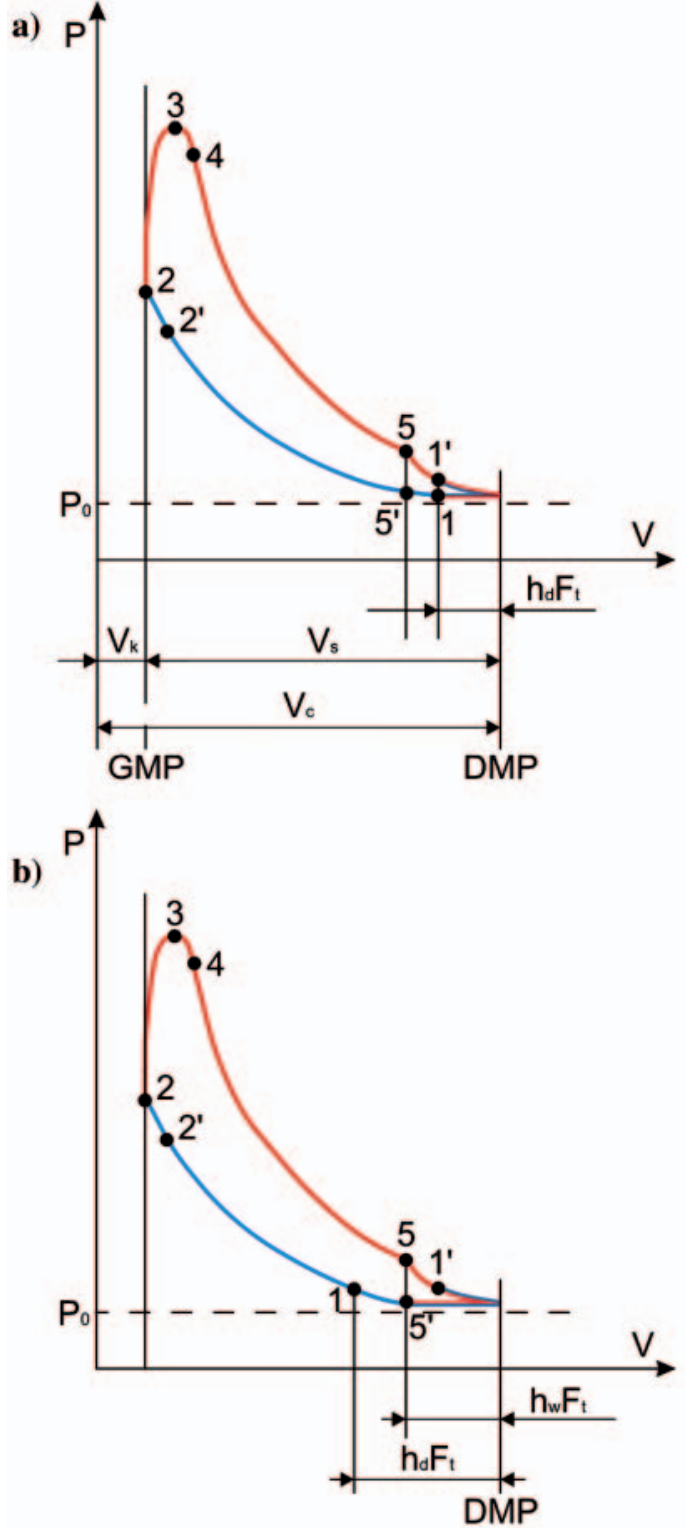

Fig. 2. Working cycles of two-stroke engines with a) uniflow scavenging, b) lateral scavenging for $\boldsymbol{h}_{\boldsymbol{d}}>\boldsymbol{h}_{w}, \mathbf{1}$-closing the air inlet ports, 5 - closing the exhaust gas exit valve (Fig. a), closing the exhaust gas outlet ports (Fig. b), 5-opening the exhaust gas exit valve (Fig. a), opening the exhaust gas outlet ports (Fig. b), 1'-opening the air admission nonreturn valves, $\boldsymbol{p}_{\text {- }}$ - ambient pressure, $\boldsymbol{V}_{\boldsymbol{t}}$ - combustion chamber volume, $\boldsymbol{V}$-displacement volume, $\boldsymbol{V}$ - total cylinder volume, $\boldsymbol{G M P}$ - upper dead centre of the piston, $\mathbf{D M P}$ - lower dead centre of the piston, $\boldsymbol{p}$-pressure, $\boldsymbol{V}$-volume

To evaluate the operation of a real engine in given conditions, as described in Ref. [2], its operation should be compared with the theoretical operation.

\section{THEORETICAL OPERATION OF INTERNAL COMBUSTION ENGINES AND ITS PRACTICAL MEANING}

In case of diesel engines, of high importance is not only the possible and needed operation, which depends on the effective work $\mathrm{L}_{\mathrm{e}}$ and time (6), but also the theoretical operation, which makes it possible to assess how far the possible engine operation differs from the standard operation. To do this, we need to know the theoretical work $\left(\mathrm{L}_{\mathrm{t}}\right)$ and the time $\tau$ of the operation. The work $\mathrm{L}_{t}$ can be easily calculated based on the average theoretical pressure $\left(p_{t}\right)$. The theoretical operation, in other words: the theoretically possible operation $\left(D_{L}\right)$ can be determined using formula (2) and taking into account relation (6) as: 


$$
D_{L_{t}}=\int_{0}^{t} L_{t}(\tau) d \tau
$$

The theoretical cycles of diesel engines include thermodynamic cycles of this type of engines, such as the Sabathe cycle and the Diesel cycle. They are used for comparing the ideal and real course of processes of thermal energy conversion into mechanical energy in arbitrary diesel engines (and for this reason are frequently referred to as the reference cycles). The theoretical Sabathe cycle is a relatively good description of the real cycles recorded in high-speed diesel engines, while the theoretical Diesel cycle well models the real cycles taking place in low-speed engines of this type [8].

The calculations of the theoretical work $\left(\mathrm{L}_{\mathrm{t}}\right)$ need the information of the work $\left(\mathrm{L}_{\mathrm{t} 1}\right)$ done during one theoretical cycle of engine operation. Unfortunately, the theoretical Sabathe and Diesel cycles cannot be directly used in this case. Instead, we should use their modified versions which take into account the specifics of operation of particular internal combustion engine types. In case of two-stroke engines with uniflow scavenging, this specific results from the delay of the outlet vale closing, which makes the fresh charge compression start not after reaching DMP by the piston, but after the exhaust gas outlet valve has been closed (Fig. 2 a, point 5'). At the same time for engines with lateral scavenging and port heights $h_{d}>h_{w}$, this specific results from the delay in closing the air inlet ports by the piston with respect to the DMP (Fig. 2b, point 1). The theoretical cycles, (Fig. 3a and Fig. 3b), in which compression of the working medium also does not begin at the DMP, but later at point 1 , reflect the specifics of the both types of engines. A separate problem is taking into account the fact that the exhaust gas decompression ends at point 5 ' of the real diagram, and not at DMP (Fig. 2).

The presented discussion shows that for low-speed engines the theoretical cycle in the form of the modified Diesel cycle additionally taking into account the isobaric heat abstraction can be used (Fig. 3a), while the cycle used for medium-speed engines is the modified theoretical Diesel cycle taking into account additional isothermal heat abstraction (Fig. 3b).

The assumption that the process along the path $5-1$ is isothermal $(\mathrm{T}=\mathrm{idem})$ can raise some doubts, as this process is difficult to execute. Moreover, we cannot determine the internal energy in this process, as for $\mathrm{T}=\mathrm{idem}$ the specific thermal capacity $\mathrm{c}_{\mathrm{T}} \rightarrow \infty$. But there is no logical, nor empirical reason for not using this process as the reference process. Its applicability is justified by the facts that:

- although in time $\mathrm{t}=\mathrm{t}_{1}-\mathrm{t}_{5}$ (when the cylinder is filled up with the fresh air) the heat is delivered to the fresh charge, which is heated by the elements composing the working space, but this time is short and the temperature does not change sufficiently to make adopting the assumption $\mathrm{T}_{5}=\mathrm{T}_{1}=\mathrm{T}=$ idem impossible. In cases when the temperature is subject to small but recordable changes we can assume that the heat supply to the fresh charge is the isothermal process taking place at a constant average temperature $\mathrm{T}_{15}$,

- since the time tof this process is short, we can assume that in that time the fresh charge mass $(\mathrm{m})$ and consequently its internal energy (U) do not change remarkably, i.e.: if $\mathrm{m}=$ idem then $\mathrm{U}=$ idem at $\mathrm{T}=\mathrm{idem}$.

A distinctive advantage of this process is that it is most economical.

For the modified Diesel cycle taking into account first the isochoric and then the isobaric heat abstraction, the theoretical work done in the cylinder during one cycle is given by the formula [7]: a)

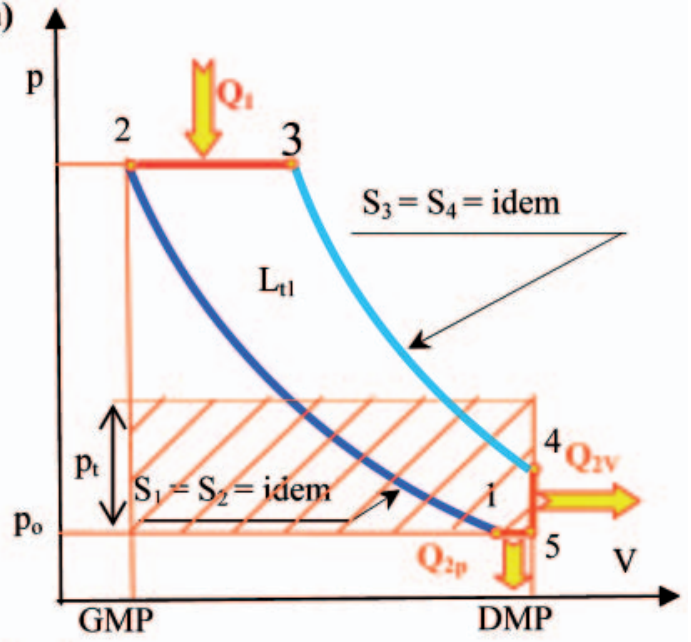

b)

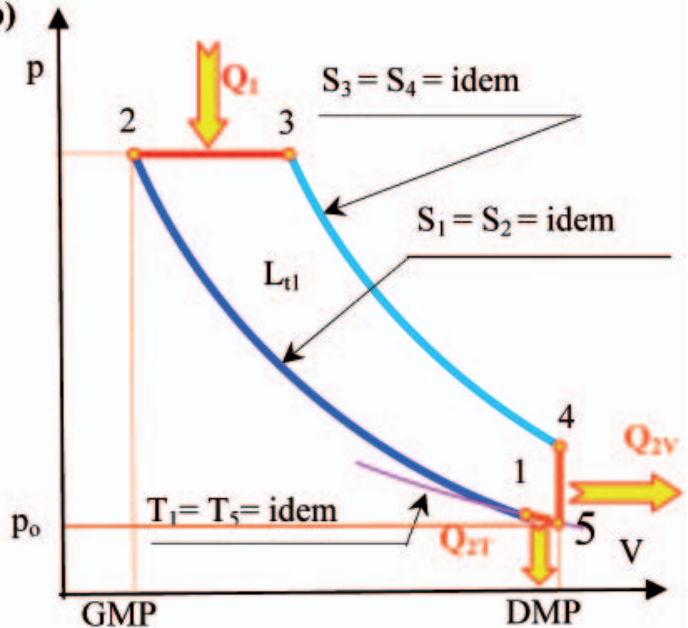

Fig. 3. Modified theoretical cycles: a) Diesel cycle taking into account isobaric heat abstraction, b) Diesel cycle taking into account isothermal heat abstraction: $\boldsymbol{L}_{\boldsymbol{t} 1}$ - theoretical work of the cycle, $\boldsymbol{p}$ - pressure,

$\boldsymbol{p}$ - average theoretical pressure, $\boldsymbol{V}$-volume, $\boldsymbol{T}$-temperature, $\boldsymbol{S}$ - entropy ${ }^{t} \boldsymbol{Q}_{1}$ - supplied heat, $\boldsymbol{Q}_{2 v}$ - heat abstracted at constant volume (isochoric), $\boldsymbol{Q}_{2 p}$ - heat abstracted at constant pressure (isobaric), $\boldsymbol{Q}_{2 T}$ - heat abstracted at constant temperature (isothermal), $\mathbf{G M P}$ and $\mathbf{D M P}$ - upper and lower dead centre of the piston, respectively

$$
\begin{gathered}
\mathrm{L}_{\mathrm{tl}}=\mathrm{Q}_{1}-\left(\mathrm{Q}_{2 \mathrm{~V}}+\mathrm{Q}_{2 \mathrm{p}}\right)= \\
=\mathrm{mc}_{\mathrm{v}}\left[\kappa\left(\mathrm{T}_{3}-\mathrm{T}_{2}\right)-\left(\mathrm{T}_{4}-\mathrm{T}_{5}+\kappa\left(\mathrm{T}_{5}-\mathrm{T}_{1}\right)\right)\right]
\end{gathered}
$$

or:

$$
\begin{gathered}
\mathrm{L}_{\mathrm{t} 1}=\mathrm{Q}_{1}-\mathrm{Q}_{2}=\mathrm{p}_{3} \mathrm{~V}_{3}+ \\
+\int_{3}^{4} \mathrm{pdV}-\left(\mathrm{p}_{5} \mathrm{~V}_{5}-\mathrm{p}_{1} \mathrm{~V}_{1}+\int_{1}^{2} \mathrm{pdV}+\mathrm{p}_{2} \mathrm{~V}_{2}\right) \\
\mathrm{p}_{1}=\mathrm{p}_{5}
\end{gathered}
$$

At the same time for the modified Diesel cycle taking into account first the isochoric and then the isothermal heat abstraction, the abovementioned theoretical work is given by the formula [7]:

$$
\begin{gathered}
\mathrm{L}_{\mathrm{t} 1}=\mathrm{Q}_{1}-\left(\mathrm{Q}_{2 \mathrm{v}}+\mathrm{Q}_{2 \mathrm{~T}}\right)= \\
=\mathrm{mc}_{\mathrm{v}}\left[\mathrm{K}\left(\mathrm{T}_{3}-\mathrm{T}_{2}\right)-\left(\mathrm{T}_{4}-\mathrm{T}_{5}\right)\right]-\mathrm{T}_{5}\left(\mathrm{~S}_{5}-\mathrm{S}_{1}\right)
\end{gathered}
$$

or:

$$
\begin{gathered}
\mathrm{L}_{\mathrm{t} 1}=\mathrm{Q}_{1}-\mathrm{Q}_{2}=\mathrm{p}_{3} \mathrm{~V}_{3}+ \\
+\int_{3}^{4} \mathrm{pdV}-\left(\int_{5}^{1} \mathrm{pdV}+\int_{1}^{2} \mathrm{pdV}+\mathrm{p}_{2} \mathrm{~V}_{2}\right)
\end{gathered}
$$


Obviously, taking into account the number of realised cycles $\mathrm{n}$ and the number of cylinders $\mathrm{k}$, the theoretical work $\left(\mathrm{L}_{\mathrm{t}}\right)$ needed for calculating the theoretical engine operation can is given by the formula [7]:

$$
\mathrm{L}_{\mathrm{t}}=\mathrm{k} \cdot \mathrm{n} \cdot \mathrm{L}_{\mathrm{t} 1}
$$

or, after calculating the average theoretical pressure $\mathrm{p}_{\mathrm{t}}$ (Fig. $4 a)$, by the formula [7]:

$$
\begin{aligned}
\mathrm{L}_{\mathrm{t}}=\mathrm{k} \cdot \mathrm{n} \cdot \mathrm{L}_{\mathrm{t} 1}=\mathrm{k} \cdot \mathrm{n} \cdot \mathrm{p}_{\mathrm{t}} \cdot\left(\mathrm{V}_{5}-\mathrm{V}_{2}\right)= \\
=\mathrm{k} \cdot \mathrm{n} \cdot \mathrm{p}_{\mathrm{t}} \cdot \Delta \mathrm{V}_{5,2}
\end{aligned}
$$

where:

$$
\begin{aligned}
& \mathrm{V}_{5}-\mathrm{V}_{1}=\Delta \mathrm{V}_{5,2}=\mathrm{V}_{\mathrm{s}} \\
& \mathrm{k}^{5} \quad-\text { number of cylinders in the engine, } \\
& \mathrm{n} \quad-\text { number of realised theoretical cycles in the operating } \\
& \text { time interval }[0, \mathrm{t}] \text {, } \\
& \mathrm{L}_{\mathrm{t} 1} \quad-\text { work of a single theoretical cycle in the cylinder of } \\
& \text { an ideal engine, } \\
& \mathrm{p}_{\mathrm{t}} \quad-\text { average theoretical pressure, } \\
& \mathrm{V}_{\mathrm{t}} \quad-\text { cylinder displacement volume, } \\
& \mathrm{V}_{5}^{\mathrm{s}}, \mathrm{V}_{2} \text { - cylinder working space volumes (having the }
\end{aligned}
$$

Comparing the effective work done by the engine in time $\mathrm{t}$ and the theoretical work which can be done by an (ideal) theoretical engine in the same time we can estimate the degree of excellence $\left(\varepsilon_{\mathrm{Ln}(\mathrm{ob})}\right)$ of energy conversion into effective work $\left(\mathrm{L}_{\mathrm{e}}\right)$ by the examined engine, which can be named the degree of excellence of engine operation. This degree can be calculated for two cases. The first case takes place when we cannot assume that during the time $t$ of engine operation the same effective work is generated in each engine cylinder. Then the degree of excellence $\left(\varepsilon_{\mathrm{Ln}(\mathrm{ob})}\right)$ of energy conversion in the engine can be given by the formula [7]:

$$
\varepsilon_{\mathrm{Ln}(\mathrm{ob})}=\frac{\sum_{\mathrm{k}=1}^{\mathrm{m}} \sum_{\mathrm{j}=1}^{\mathrm{n}} \mathrm{L}_{\mathrm{e}(\mathrm{j}) \mathrm{k}}}{\mathrm{L}_{\mathrm{t}}}
$$

where:

$\mathrm{L}_{\mathrm{e}(\mathrm{j}) \mathrm{k}}$ - effective work of the $\mathrm{j}$-th cycle in the $\mathrm{k}$-th engine cylinder,

$\mathrm{L}_{\mathrm{t}} \quad$ - theoretical work calculated using formula (13) for the modified Diesel cycle.

The degree of excellence of energy conversion into work, defined by formula (14), describes haw far the energy conversion into work in the real engine differs from the theoretical work of an ideal engine, after $n$ cycles.

The second case takes place when we can assume that in time t of engine operation the same effective work is generated in each cylinder. Then the abovementioned degree of excellence $\left(\varepsilon_{\mathrm{Ln}(\mathrm{ob})}\right)$ can be calculated using formula [7]:

where:

$$
\varepsilon_{\mathrm{Ln}(\mathrm{ob})}=\frac{\mathrm{L}_{\mathrm{e}}}{\mathrm{L}_{\mathrm{t}}}=\frac{\mathrm{L}_{\mathrm{el}}}{\mathrm{L}_{\mathrm{t} 1}} ; \mathrm{L}_{\mathrm{e}}=\mathrm{knL}_{\mathrm{e} 1}
$$

$\mathrm{k} \quad-$ number of engine cylinders,

$\mathrm{n} \quad-$ number of realised theoretical cycles in the operating time interval $[0, \mathrm{t}]$,

$\mathrm{L}_{\mathrm{e} 1}$ - effective work of a single real cycle in the engine cylinder.

Of some interest is how far the operation of the real engine as a whole differs from the operation of an ideal engine. Analysing the same cases as for the engine degree of excellence $\left(\varepsilon_{\mathrm{Ln}(\mathrm{ob})}\right)$ of energy conversion into effective work $\left(\mathrm{L}_{\mathrm{e}}\right)$, we can define the degree of engine operation excellence $\left(\varepsilon_{\mathrm{Dn}(\mathrm{ob})}\right)$. In the first case when we have to assume that different effective work is generated in each cylinder in particular time intervals $t_{(\mathrm{i}) \mathrm{k}}$ composing the overall time $t$ of engine operation, the degree of engine operation excellence $\left(\varepsilon_{\mathrm{Dn}(\mathrm{ob})}\right)$ can be calculated as [7]:

$$
\varepsilon_{\text {Dn }(o b)}=\frac{\sum_{k=1}^{m} \sum_{j=1}^{n} L_{e(j) k} t_{(j) k}}{L_{t} t}
$$

where:

$\mathrm{L}_{\mathrm{e}(\mathrm{i}) \mathrm{k}}$ - effective work of the $\mathrm{j}$-th cycle in the $\mathrm{k}$-th engine cylinder,

$\mathrm{t}_{(\mathrm{j}) \mathrm{k}} \quad$ - time of generation of work $\mathrm{L}_{\mathrm{e}(\mathrm{j}) \mathrm{k}}$,

$\mathrm{L}_{t}^{(j) \mathrm{k}} \quad-$ theoretical work calculated using formula (13) for the modified Diesel cycle.

In the second case when we can assume that in the time $t$ of engine operation the same effective work $\mathrm{L}_{\mathrm{e} 1}$ is generated in each cylinder, the excellence degree $\left(\varepsilon_{\mathrm{Dn}(\mathrm{ob})}\right)$ can be expressed using the same relation as the degree of excellence $\left(\varepsilon_{\mathrm{Ln}(\mathrm{ob})}\right)$ of energy conversion into effective work $\left(\mathrm{L}_{\mathrm{e}}\right)$ by the examined engine (15). Consequently:

$$
\sum_{\mathrm{i}=1}^{\mathrm{n}} \mathrm{t}_{(\mathrm{j}) \mathrm{k}}=\mathrm{t}
$$

Therefore we can assume that in the second case the excellence degree $\left(\varepsilon_{\mathrm{Dn}(\mathrm{ob})}\right)$ of operation of an internal combustion engine is equivalent to the excellence degree $\left(\varepsilon_{\mathrm{Ln}(\mathrm{ob})}\right)$ of energy conversion into effective work $\left(\mathrm{L}_{\mathrm{e}}\right)$ in this engine.

If we take into account the indicated work $\mathrm{L}_{\mathrm{i}}$ in these analyses, we can determine the coefficient (degree) of engine operation dissipation, caused by the energy lost for overcoming mechanical resistances. The abovementioned degree of engine operation dissipation could be defined as [7]:

$$
\xi_{D n(o b)}=\frac{\sum_{k=1}^{m} \sum_{j=1}^{n} L_{e(j) k} \mathrm{t}_{(j) k}}{\sum_{k=1}^{m} \sum_{j=1}^{n} L_{i(j) k} t_{(j) k}}
$$

If we can assume that in the time $t$ of engine operation the case takes place that: $\mathrm{L}_{\mathrm{i}}=\mathrm{idem}$ and $\mathrm{L}_{\mathrm{e}}=$ idem, then the engine operation dissipation degree is equal to its mechanical efficiency.

Formulas (9) and (11) can be only used when the paths of thermodynamic processes of the cycles are known. On the other hand, when we know the functional relations between the pressures and the volumes which are characteristic for these processes, the above formulas take the forms:

$$
\begin{aligned}
& \mathrm{L}_{\mathrm{t} 1}=\mathrm{Q}_{1}-\mathrm{Q}_{2}=\mathrm{p}_{3} \mathrm{~V}_{3}+ \\
& +\int_{V_{3}}^{\mathrm{V}_{4}} \mathrm{p}(\mathrm{V}) \mathrm{dV}-\left(\mathrm{p}_{5} \mathrm{~V}_{5}-\mathrm{p}_{1} \mathrm{~V}_{1}+\int_{\mathrm{V}_{1}}^{\mathrm{V}_{2}} \mathrm{p}(\mathrm{V}) \mathrm{dV}+\mathrm{p}_{2} \mathrm{~V}_{2}\right) \\
& \mathrm{L}_{\mathrm{t} 1}=\mathrm{Q}_{1}-\mathrm{Q}_{2}=\mathrm{p}_{3} \mathrm{~V}_{3}+ \\
& +\int_{V_{3}}^{\mathrm{V}_{4}} \mathrm{p}(\mathrm{V}) \mathrm{dV}-\left(\int_{\mathrm{V}_{5}}^{\mathrm{V}_{1}} \mathrm{p}(\mathrm{V}) \mathrm{dV}+\int_{\mathrm{V}_{1}}^{\mathrm{V}_{2}} \mathrm{p}(\mathrm{V}) \mathrm{dV}+\mathrm{p}_{2} \mathrm{~V}_{2}\right)
\end{aligned}
$$

The above analysis shows that in the case when the functional relations $p=f(V)$ for the examined processes are known, formula (18) can be used instead of formula (9) and (19) instead of (11). 


\section{REMARKS AND CONCLUSIONS}

- The operation of the internal combustion engine was interpreted as the delivery of the required energy in given time, which can be expressed in the form of a physical quantity having the measure unit called joule-second.

- When discussing energy related advantages of internal combustion engines, we should analyse their operation and not only their work. Beside the work itself, the operation analysis takes into account also time of its realisation.

- The proposed method can be applied for calculating the theoretical standard operation, which enables comparison between different real diesel engines revealing different levels of wear. The theoretical work needed for calculating this operation was calculated based on the modified theoretical Diesel cycles, different for different engine rotational speeds. The modification introduced in those cycles consisted in additional taking into account the heat abstraction according to the isobaric or isothermal processes. For low-speed engines the additional heat abstraction modelled as the isobaric process was proposed, while that in the medium-speed engines was assumed to take place according to the isothermal process.

- The presented analysis reveals that it is not advisable to use traditional and well-known theoretical Diesel cycles for determining the theoretical work making the basis for calculating the engine operation value.

- The article presents the deterministic method applied for evaluating the theoretical operation of the piston internal combustion engine used as the main engine on a ship, but this method can be applied to any piston internal combustion engines.

- A separate problem is developing a method for assessing the operation of internal combustion engines in the stochastic formulation. In this case we should take into account that the energy conversion processes taking place during engine operation have stochastic nature.

- A similar analysis can be performed for four-stroke internal combustion engines, and similar formulas can be derived for determining their theoretical operation.

- The article also illustrates the applicability of the assessment of theoretical internal combustion engine operation, done in the above way, for evaluating real operations of this type of internal combustion engines.

\section{BIBLIOGRAPHY}

1. Aeberli K.: New high-economy engines for panama containerships and large tankers, Wartsila Switzerland Ltd, Winterthur, September 2007
2. Girtler J.: Work of a compression-ignition engine as the index of its reliability and safety. II International ScientificallyTechnical Conference EXPLO-DIESEL \& GAS TURBINE"01. Conference Proceedings. Gdansk-Miedzyzdroje-Copenhagen, 2001, pp.79-86

3. Girtler J.: Possibility of valuation of operation of marine diesel engines. Journal of POLISH CIMAC, Vol. 4, No 1, 2009

4. Girtler J.: Energy-based aspect of operation of diesel engine. COMBUSTION ENGINES (Silniki Spalinowe) No 2/2009 (137)

5. Girtler J.: Conception of valuation of combustion engine operation. Journal of KONES. Powertrain and Transport. Editorial Office Institute of Aeronautics BK, Warsaw 2008

6. Girtler J.: A method for evaluating the performance of a marine piston internal combustion engine used as the main engine on a ship during its voyage in different sailing conditions. Polish Maritime Research. Vol. 17, iss. 4(67), 2010

7. Girtler J.: Possibility of defining theoretical operation for diesel engines in energy terms. COMBUSTION ENGINES (Silniki Spalinowe) Nr 3/2011(146). Abstract p. 62. Full text CD: PTNSS 2011-SD-005

8. Łosiewicz Z.: Probabilistic diagnostic model of the marine main engine (in Polish). Ph.D. thesis, Faculty of Ocean Engineering and Ship Technology, Gdansk University of Technology, Gdansk

9. Piotrowski I., Witkowski K.: Operation of marine internal combustion engines (in Polish). AM, Gdynia 2002

10.Piotrowski I., Witkowski K.: Marine internal combustion engines (in Polish). Wyd. TRADEMAR, Gdynia 1996

11. Rosłanowski J.: Identification of ships propulsion engine operation by means of dimensional analysis. Journal of POLISH CIMAC, Vol. 4, No 1, 2009

12.Rudnicki J.: Loads of ship main diesel engine in the aspect of practical assessment of its operation. Journal of POLISH CIMAC, Vol. 3, No 1, 2008

13.Rudnicki J.: On making into account value of operational applied to ship main propulsion engine as an example. Journal of POLISH CIMAC, Vol. 4, No 1, 2009

14.Wajand J.A., Wajand J. T.: Medium- and high-speed internal combustion engines (in Polish). WNT, Warszawa 2005

15.Wojnowski W.: Marine diesel engine power plants. (in Polish) Pt. I. Wyd. AMW, Gdynia 1998.

\section{CONTACT WITH THE AUTHOR}

Jerzy Girtler, Prof.

Faculty of Ocean Engineering and Ship Technology

Gdansk University of Technology Narutowicza 11/12

80-233 Gdansk, POLAND

tel.: (+48 58) 347-24-30; fax: (+48 58) 347-19-81 e-mail: jgirtl@pg.gda.pl 